\title{
Single-trial Classification of Viewed Characters using Single-channel EEG Waveforms
}

\author{
Minoru Nakayama, Hiroshi Abe \\ Tokyo Institute of Technology \\ Ookayama, Meguro, Tokyo 152-8552 Japan
}

\begin{abstract}
Electroencephalograms (EEGs) and Eventrelated potentials (ERP) have long been used to observe the human visual perception process, such as the human response to letters, Kanji characters and symbols. This paper examines the possibility of classifying characters when viewed by subjects in single trials using single-channel EEG waveforms of the frontal area $(\mathrm{Fz})$ and the occipital area $(\mathrm{Oz})$ of the brain. The first 20 trials for each character were used for calibration, and the remaining trials were assigned to the test data set. Feature vectors for each trial were created as EEG waveforms from 100 up to 800 msec. after the stimuli was shown. To extract features of waveforms, the regression relationship between EEG and ERP waveforms was used to transform observed signals. As a result, the performance of cross validation rates of the test data set increased incrementally during the perceptual process, for both $\mathrm{Fz}$ and $\mathrm{Oz}$, when the predicted waveforms were measured using the regression relationship. Also, the effectiveness of the prediction using the regression relationship for the classification performance of viewed characters was determined during the perceptual process. This provides evidence that a procedure using the relationship between EEG and ERP is effective in predicting viewed characters.
\end{abstract}

\section{Introduction}

In the Digital Revolution, information has been provided using many kinds of displays, computer screens being one example. To help people perceive information from displays, the determination of whether or not they can recognize characters such as symbols or letters is required as a basic part of the support system. Therefore, some techniques related to a Brain Machine Interface (BMI), which can be used to observe viewer's recognition of characters, have been studied [9]. For BMI research, equipment such as fMRI or MEG are used to observe human brain activity, and electroencephalograms (EEGs) also provide significant information [7].
EEGs have been used to observe the human visual perception process, such as its response to visual stimulus, and have also been used to detect mental activities such as in a BMI or in a Brain Computer Interface (BCI) [3]. EEGs are noisy data because a single stimulus point at the scalp is quite small (5-10 $\mu V)$. Therefore some additional information, such as references for the signals, is often required to extract information which is significant from single-trial EEGs [12]. A conventional technique for reducing noise on EEGs is the use of event-related potentials (ERPs) [14]. To extract an ERP waveform, EEG waveforms which record responses to stimulus need to be averaged as much as is possible. As ERPs can indicate some feature of events, they may be used as a reference to extract distinct information from single-trial EEGs. The relationship between EEGs and ERPs is simple, but ERPs as a prototypical signal can be a key piece of information for the understanding of single trial EEG waveforms. This means that every EEG waveform of a visual stimulus contains an ERP waveform with some artifacts, and an appropriate signal processing method is required to reduce these artifacts. If the appropriate ERPs are extracted from a small number of trials, a reference can be created during EEG measurement of the calibration process. This hypothesis has been supported by a preliminary simulation experiment [10]; the most effective procedure for doing this is still unclear.

The aim of this paper is to examine the performance of character classification of symbols or Kanji characters viewed by subjects, using singlechannel single-trial EEGs in response to the perceptual process. This process takes into consideration the activities in the frontal and occipital areas of the human brain. The effectiveness of a signal prediction technique with Support Vector Regression (SVR) [16] using a relationship which is based on the regression between EEG and ERP was measured. In particular, the possibility of classification using single-channel EEG waveforms will be addressed. The overall performance was evaluated for its accuracy and the performance changes were discussed, with adequate consideration given to the mental chronological process. The 


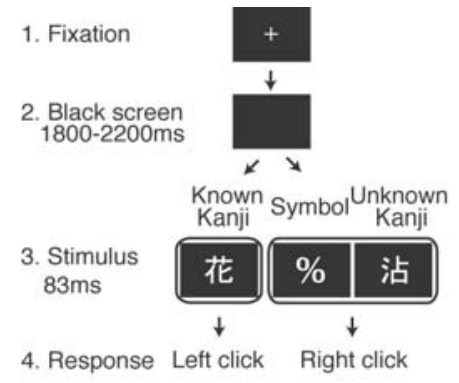

(a)

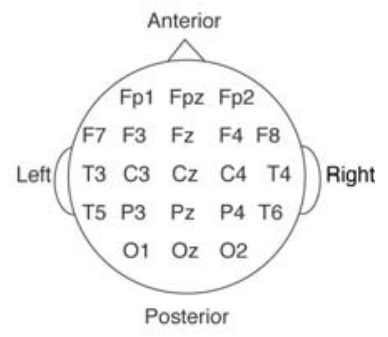

(b)

Figure 1. Experimental task for Kanji recognition (a) and placement of electrodes (b)

degree of individual differences was also considered to determine the possibility of applying this technique to the classification of viewed characters.

\section{Method}

In this section, new procedures for estimating viewed characters observed by users using single channel and single-trial EEG waveforms are summarized.

\subsection{EEG/ERP waveforms}

To observe EEG waveforms during the character perception process, a Kanji recognition task was conducted [1, 2].

2.1.1. Kanji recognition Task. The experimental task is depicted in Figure 1(a). When a Kanji character or a symbol was flashed on a computer screen, EEG waveforms were recorded from 21 electrodes, as shown in Figure 1(b). This experiment examined the Kanji recognition process in accordance with the differences in ERP waveforms among Kanji which were known, Kanji which were unknown, and symbols. The number of trials for each kind of stimuli was 100 . For each subject, a total of 200 trials were analyzed. The procedure was as follows: (1) The subject clicked the left button of a mouse to start the trial. (2) A black screen which gave a random delay of $1.8-2.2 \mathrm{sec}$. was then presented. (3) An image of a character (either a known Kanji, an unknown Kanji, or a symbol) was presented briefly (83 msec.). (4) The subject had to report if the image presented was a known Kanji or unknown Kanji by clicking the mouse's left or right button respectively. Four subjects who were 23-25 years old participated in this experiment.

2.1.2. EEG/ERP Recording. The EEGs were recorded from 21 scalp electrodes which included Fpz, Oz, and 19 other locations, according to the international 10-20 system, using an electrocap

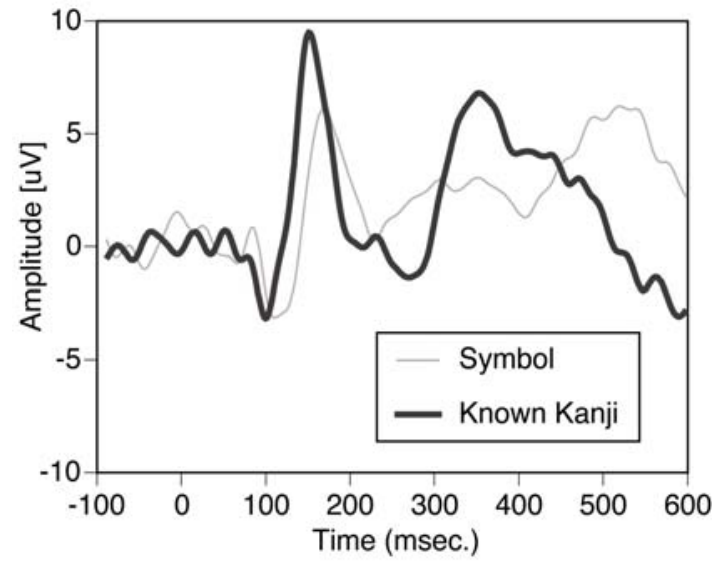

Figure 2. ERP waveforms of symbol and Kanji character stimuli from the $\mathrm{Fz}$ electrode

manufactured by Electro-cap International. A ground electrode was placed on the forehead. All scalp electrodes were referenced to the subject's ear lobes. The signals were amplified and $0.5-100 \mathrm{~Hz}$ band pass filtered using a BIOTOP amplifier from NEC. The signals were stored in a PC that had a $512 \mathrm{~Hz}$ sampling rate. After being recorded, the signals were digitally filtered to reduce line noise below $40 \mathrm{~Hz}$. Trials containing an EOG amplitude of more than 70 $\mu V$ were removed from the subsequent analyses, because of the large artifactual influences of eye movements on the EEGs [8]. Each subject's single trial raw EEGs were recorded from every electrode, from $100 \mathrm{msec}$. before to $1000 \mathrm{msec}$. after stimulus onset in all correct trials. Baseline voltages were subtracted from the raw data of these EEGs. As a baseline for each trial and for each electrode, the average voltage in the preceding $100 \mathrm{msec}$. interval before stimulus onset during the trial was used. Correct trials were trials in which the subject correctly discriminated between the characters or symbols presented. 


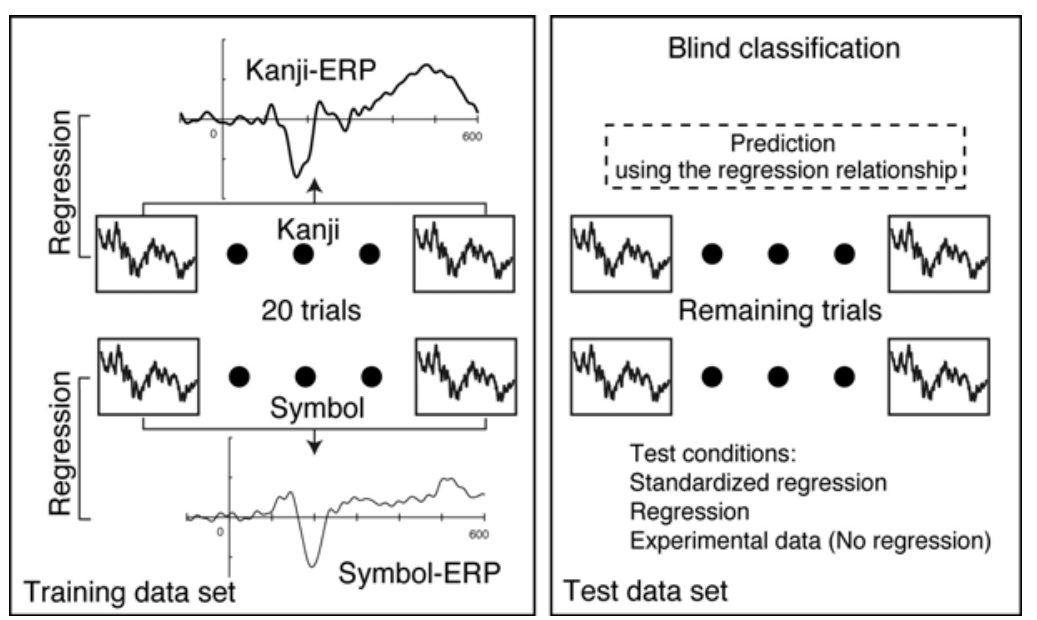

Figure 3. EEG signal processing diagram

ERP waveforms reflect the kind of characters that were shown [1, 2]. Figure 2 shows an ERP extracted from the Fz electrode. The waveforms of all correct responses were summed up, and compared with ERP waveforms of symbols and Kanji. These ERP waveforms are well smoothened and show the differences in response to the stimuli. Some significant differences between symbols and Kanji were observed in P100 (at 100 msec.), N170 (at 170 msec.) and P250 (at 250 msec.) on Fz and Oz. Mean reaction time was around 500 msec., and the waveforms between 100 and 500 msec. were significantly different because the recognition processes were different.

In this paper, we focused on single-channel EEG waveforms for symbols and known Kanji measured at the $\mathrm{Oz}$ and $\mathrm{Fz}$ electrode, because the occipital area reflects visual perception and the frontal area reflects the discrimination process. The differences in waveforms are evaluated using signals from electrodes near each area of the brain.

\subsection{Classification of single-trial EEGs}

The classification performance for single-trial EEG waveforms from the $\mathrm{Oz}$ and $\mathrm{Fz}$ electrodes were evaluated using the diagram in Figure 3. Therefore, only trials with correct responses were selected. Rates of correct selection averaged over 90\% [1]. All EEG waveforms for each subject were smoothened in advance, to permit the application of some signal processing. The first 20 trials, which showed both symbols and Kanji, were used to create a training data set for each subject. A test data set was then created using the remainder of the data.

2.2.1. Classification without predictions. For the training data (the left side of Figure 3), classification labels for symbols or Kanji were given to each data set. These data sets contained significant response periods, which were controlled to be from 100-150 (for 26 data points) to 100-500 msec. (for 205 data points) long. The duration was extended step by step, by 100 msec. up to 500 msec. From $500-800$ msec. (for 153 data points) data was collected because the response decision was made.

Here, $x_{i}$ is defined as an EEG potential, $t$ is defined as the stimulus given, and the training data set comprises $N$ input vectors $\mathbf{x}=\left(x_{1}, \ldots, x_{N}\right)$, with corresponding target values: $t \in\{-1:$ symbol, $+1:$ Kanji $\}$. The acquired data can be noted as $(\mathbf{x}, t)$ for each trial. A sign function, based on the SVM function, is defined as function $G$ using the Gaussian kernel. For every interval, the parameters for the Gaussian kernel were optimized using a software tool [4]. The classification was conducted using SVM with Gaussian kernel, such as LIBSVM [4].

After the training procedure was completed, the test data set was classified (the right side in Figure 3). The prediction class $\hat{t}$ is given as $\hat{t}=G(\mathbf{x})$ for the test set, and the rates for correct prediction $(t$ vs. $\hat{t})$ are evaluated across the duration of the interval. This classification procedure is called "NO-REG" in response to the following procedure.

2.2.2. Classification with ERP references. To extract the relationship between EEG and ERP, ERPs for symbols and Kanji respectively were extracted from the training data set of each subject. Because the training data set consists of trials which show both symbols and Kanji, the relationship is formulated as a regression function across both EEG responses. Therefore, the regression prediction from a single-trial EEG waveform may reflect both ERP features of symbols and Kanji. To predict an appropriate signal in response to ERP waveforms from observed EEG signals, a regression function 
from a single-trial EEG to ERP has been created, using the SVR technique. This regression training

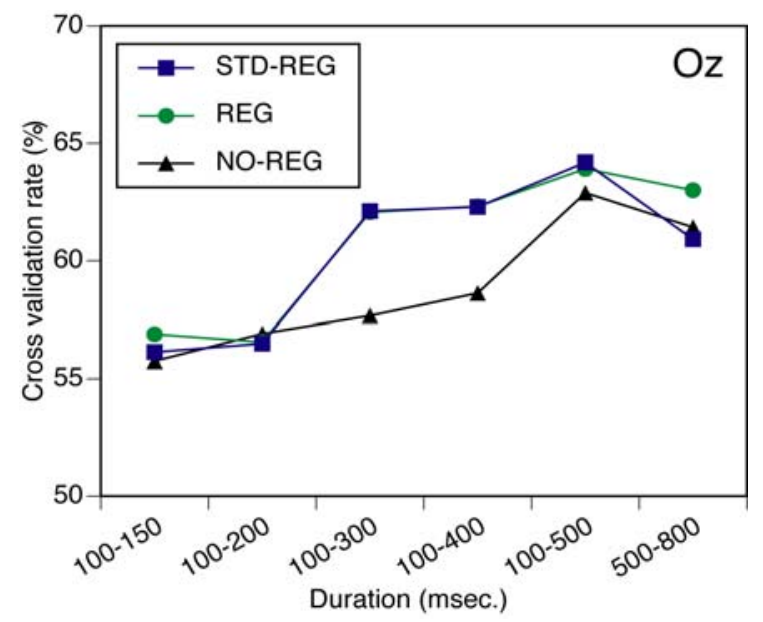

Figure 4. Cross validation rate $(\mathrm{Oz})$

procedure was conducted on a training data set, which consisted of EEGs of both symbols and Kanji for each subject.

Two prediction procedures were created in this study, as follows:

1. Regression prediction (REG)

All data were based on raw signal data. The regression function as $f$ was created for the training set. Here, $x_{i}$ is defined as EEG potentials, and $y_{i}$ is defined as an ERP which sums up EEG waveforms of all trials in the training data set. The estimated ERP $\hat{y}_{k}$ for the empirical potential $y_{k}$ at the time position $k$ is reproduced from time series data samples comprising 10 input vectors $\mathbf{x}_{k}$, so that $\mathbf{x}_{k}=\left(x_{k-9}, \ldots, x_{k}\right), \hat{y}_{k}=f\left(\mathbf{x}_{k}\right)$. The parameters of SVR were given as a standard deviation for Gaussian kernel and as a width of error pipe (epsilon: eps = 0.5) using SVMTorch [6, 5].

The relationship between the viewed character class $t$ and the estimated ERP $\hat{y}$ also can be noted as $(t, \hat{y})$ for each trial, in both the training and test data sets. Both classification training and testing use the same procedures, and use the above classification without predictions as well. This procedure can be applied to online processing. Once the regression and classification functions have been created from the training data set, EEG waveforms for consequent trials are estimated and the character believed to have been viewed is predicted, step by step.

\section{Standardized regression (STD-REG)}

All EEG waveform data for training and test data sets are standardized using the overall means and
SDs, after all trials have been completed. The remaining processing procedure is the same as

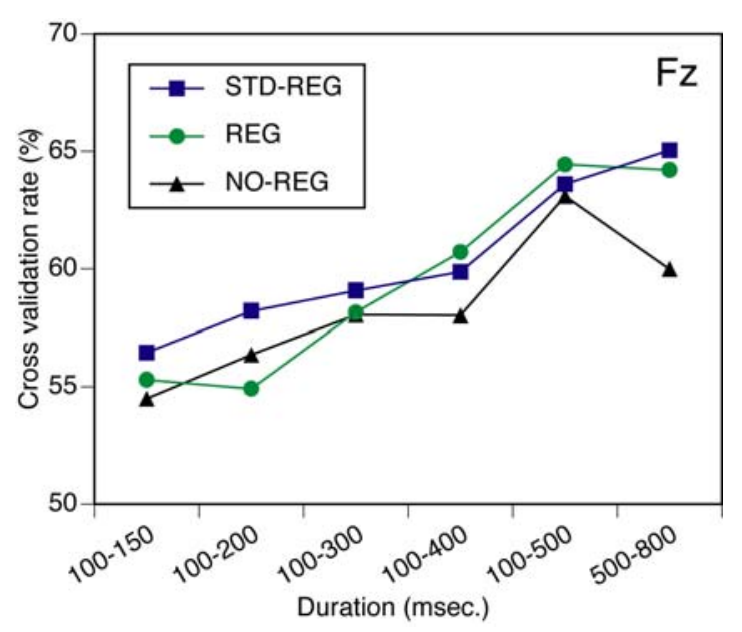

Figure 5. Cross validation rate (Fz)

"REG". This procedure can not be applied to any novel trials. This post processing may neglect the differences between trials for some subjects. Therefore, this processing can be referred to as an off-line classification.

\section{Results}

The performance of cross validation rates of the test data set and the effectiveness of the prediction using the regression relationship for classification performance were determined during the perceptual process.

\subsection{Cross validation rate}

The ability of classifying the test data was assessed as a cross validation rate using LIBSVM [4]. In this procedure, the test data set was randomly divided into 5 blocks and the performance of classifications was assessed. The rates were calculated for each subject and the mean rates across the three classification procedures were summarized.

The results for the $\mathrm{Oz}$ electrode are summarized in Figure 4. The horizontal axis shows the duration and the vertical axis shows the cross validation rate. The "NO-REG" shows the results using raw observation data. The rate increases gradually with the extension of the duration, and the highest rate is during a duration of $100-500 \mathrm{msec}$. This rate is significant using a non-parametric test [15], but other rates are not significant except in a condition with a duration of 500-800 msec. When predicted EEG waveforms were applied to classifications such as "STD-REG" and "REG", all rates were significant except for conditions with a duration of 100-150 and 


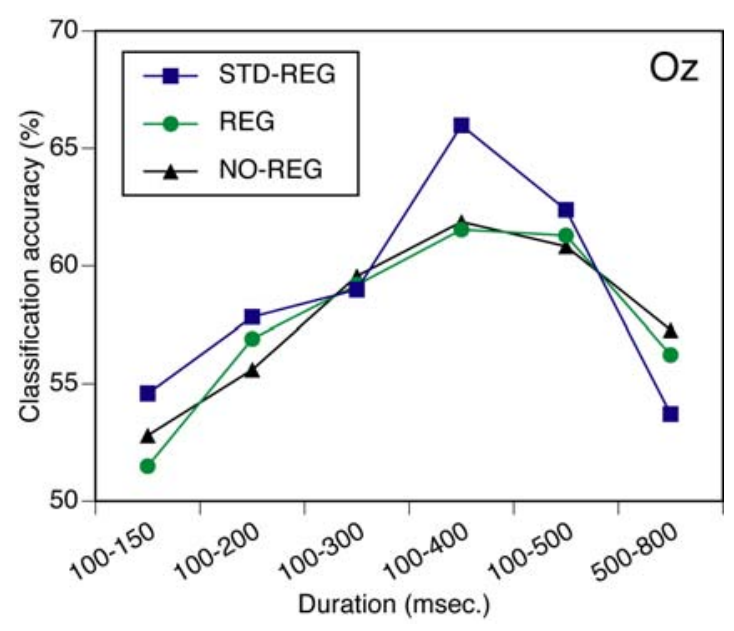

Figure 6. Classification accuracy $(\mathrm{Oz})$

100-200 msec. In particular, data for a duration of 100-300 msec. became significant when the estimated EEG waveforms were applied using the regression function. This duration includes P100, N170 and P250, so that predictions using the regression relationship between EEG and ERP may emphasize the differences in waveforms. This also suggests that the data for a duration of 100-200 msec. is insufficient for its classification, however.

The results for Fz electrodes are summarized in Figure 5. The highest performance classification was for a duration of 100-500 msec. across the three processing conditions. For this duration, "STDREG" is the highest, and "REG" is higher than "NOREG". The performance with predictions using the regression relationship is almost always higher than the performance with raw observed data across all durations. For durations up to 400 msec., all rates are not significant, however the rate with predictions is almost always higher.

In comparing the results for $\mathrm{Oz}$ and $\mathrm{Fz}$, the duration for the highest rates are different. For the $\mathrm{Oz}$ classification, predicted waveforms up to 300 msec. are sufficient, and predicted waveforms up to 500 msec. are required for $\mathrm{Fz}$ classification. Both classification results suggest that EEG waveforms before the button was pressed to make a selection (around 500 msec.) reflect the viewed characters specifically. From this, it can be determined that single-trial EEG waveforms contain significant information in response to viewed characters.

In the general visual information processing theory, the retinal image is mapped in the primary visual cortex, which is located in the occipital area [13]. During the decision task, some activations may be observed on the frontal cortex, which is located in the frontal area. Some time is needed to recognize a character which is shown, resulting in the highest peak of the cross validation rate being delayed until perception has occurred. The greater delay in

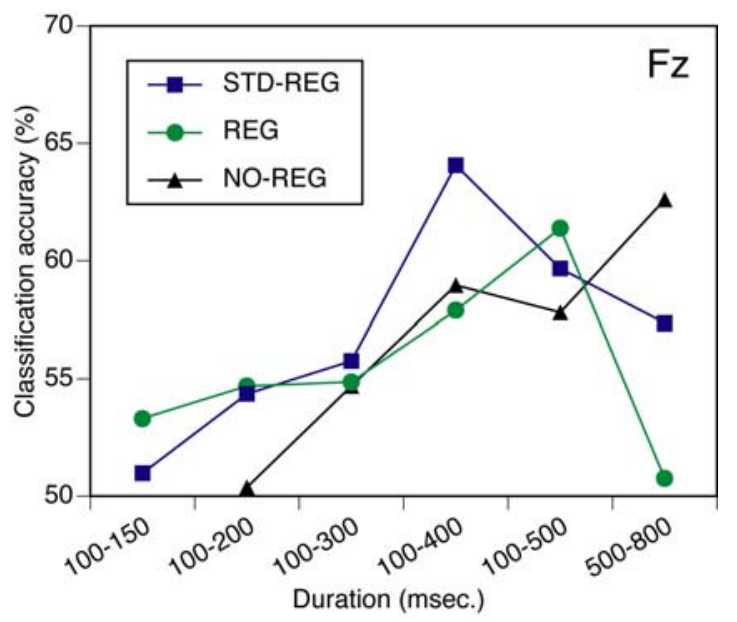

Figure 7. Classification accuracy (Fz)

perception by $\mathrm{Fz}$ as compared to $\mathrm{Oz}$ coincides with the chrological model, but the measured time of the delay seems longer than in theory. Therefore, some factors may influence the cross-validation rates in addition to influencing the image processing pathway. For Fz classification with prediction using waveforms up to $200 \mathrm{msec}$., the improvement of rates may suggest that some features of waveforms have been emphasized by the relationship between EEG and ERP even in the earlier part of the period prior to recognition, though the rate is not significant.

These results suggest that predictions using the relationship between EEG and ERP are effective.

\subsection{Classification accuracy}

The classification performance for the $\mathrm{Oz}$ electrode is summarized in Figure 6. Again, the classification model was prepared using the training data set which consisted of the first 20 trials. The model was then applied to the test data set. The rates of classification accuracy increase from the duration of $100-150$ msec. to $100-400$ msec., and the rates for $100-400$ msec. are the highest. The rates for both 100-400 msec. and 100-500 msec. are significant. During these periods, the rate for "STD-REG" is the highest across the three conditions. The regression processing of the raw data may not be effective for the classification of $\mathrm{Oz}$ waveforms. The standardized processing has a significant effect on the duration from 100-400 msec. as the rate has improved dramatically. This suggests that the deviation of waveforms influences the rate.

Fz classification performance is summarized in Figure 7. The highest levels of classification accuracy are different across the three conditions: 


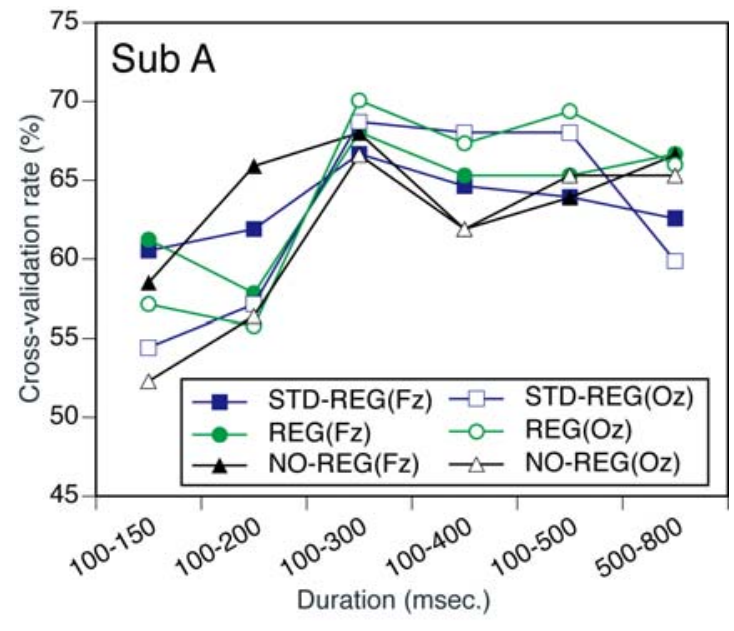

Figure 8. Cross-validation rates for $\mathrm{Oz}$ and Fz (Subject A)

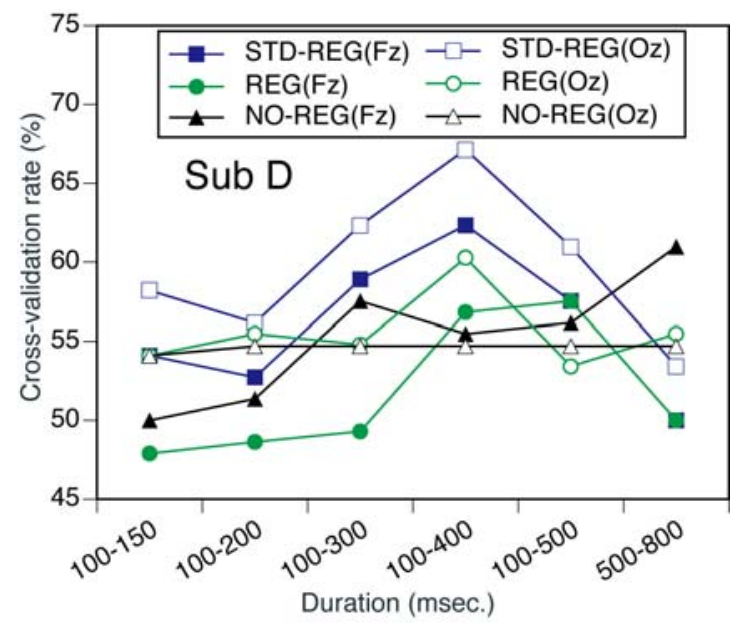

Figure 9. Cross-validation rates for $\mathrm{Oz}$ and Fz (Subject D)

100-400 msec. for "STD-REG", 100-500 msec. for "REG", and 500-800 msec. for "NO-REG". When the classification was conducted using standardized and predicted waveforms, earlier waveforms of between 100 and 400 msec. are effective. For predicted waveforms without standardization, significant classification is possible using 100-500 msec. waveform data. This result suggests that classification is impossible when using raw data from before the decision response (around 500 msec.).

In comparing results for $\mathrm{Oz}$ and $\mathrm{Fz}$, the highest rates for the duration coincide with 100-400 msec. for the "STD-REG" condition. For other conditions, the higher rate durations of $\mathrm{Fz}$ occur later than for the results of $\mathrm{Oz}$. According to the results of the cross validation rate calculations, some delay in the duration for the highest rates is expected. It is impossible to explain the differences across processing conditions for $\mathrm{Fz}$ using classification accuracy. To obtain high classification performance, the features of waveforms should be similar between the training and test data sets. In this paper, the first 20 trials were assigned to the training data set, but the consistency for features of EEG waveforms in the two data sets was not determined. Also, the preparation procedure for the training data set should be taken into consideration.

These will be the subjects of our further study.

\subsection{Individual differences in performance}

The cross-validation performances for estimating viewed characters using EEG waveforms at electrodes $\mathrm{Oz}$ and $\mathrm{Fz}$ were determined, and the feasibility was confirmed. In this subsection, the validity of the procedure will be discussed. In particular, it is necessary to determine whether the procedure can be applied to EEG waveforms of any subject. Also, the procedure depends on two sets of data, the training and test data sets, and the relationship between them may reflect classification performance. These points are discussed in two subsections.

The average performances of cross-validation rates for both $\mathrm{Oz}$ and $\mathrm{Fz}$ are significant. There may be some differences among subjects, however, because EEG waveforms are one of many kinds of human biological information, and the observed signals may be influenced by various conditions.

To discuss the differences in performance, the results of two subjects, one with good performance and one with poor performance, are compared. The individual results of cross validation rates for $\mathrm{Oz}$ and Fz are shown in Figure 8 for Subject A, and Figure 9 for Subject D.

In Figure 8, most rates for Subject A after the duration 100-200 msec. are significant, and even the rates for NO-REG at both $\mathrm{Oz}$ and $\mathrm{Fz}$ are significant for the duration, while rates for both REG and STDREG are almost always higher than the ones for NOREG. Also, rates for $\mathrm{Oz}$ are almost always higher than the ones for $\mathrm{Fz}$ for the duration. The effectiveness of the prediction using the relationship between EEG and ERP is reconfirmed. Therefore, the results of Subject A emphasize the averaged results because the tendencies mentioned above are similar to the overall results. Also, the mean rates of Subject A are higher than the overall average.

In Figure 9, most rates for Subject D, including those for REG processing, stay at the level of chance except for the rates for STD-REG during the duration from $200 \mathrm{msec}$. to $500 \mathrm{msec}$. for $\mathrm{Oz}$ and the rates for STD-REG during 100-400 msec., which are significant. According to the results for Subject D, the effectiveness of STD-REG processing is confirmed, while the effectiveness of REG processing is not observed. The STD-REG 
processing introduced a standardization for the

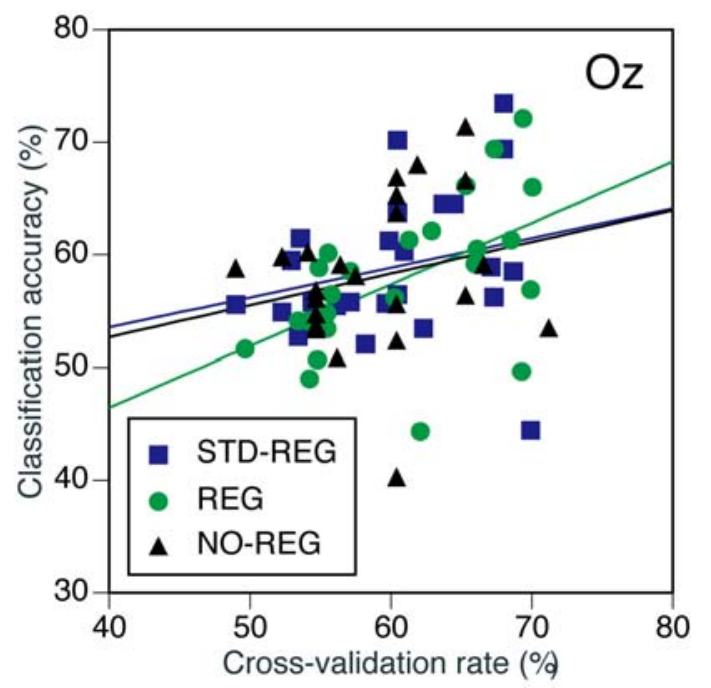

Figure 10. Relationship between crossvalidation rate and classification accuracy

(Oz)

training and test data sets. Therefore, the levels of EEG data for Subject D were different between the training and testing data sets. Also, observed EEG levels are different amongst trials. The effectiveness of STD-REG processing is confirmed for Subject D. This phenomenon suggests that while appropriate signal processing can extract some features for viewed characters from EEG waveforms, other techniques should be applied to obtain better performance. This point will be a subject of our further study.

\subsection{Relationship between cross-validation rate and classification accuracy}

In this study, the procedure for classification of viewed characters is proposed and illustrated in Figure 3, which consists of a training set of the first 20 trials and a test set of the remainder of the data. The classification performance may depend on the ability to classify the data as cross-validation rates.

The performances of cross-validation rates and classification accuracy are evaluated separately in the results section. In this section, the relationships between cross-validation and classification accuracy are discussed because the classification accuracy may depend on the ability to classify data.

In Figure 10, the relationship between the crossvalidation rate and the classification accuracy using individual data over all durations for $\mathrm{Oz}$ is summarized for each processing method, such as STD-REG, REG and NO-REG. The regression lines for each processing method overlap in the figure. Some correlationships are observed in this figure. Also, the relationship for $\mathrm{Fz}$ is summarized using the same format as in Figure 11. In comparing the two

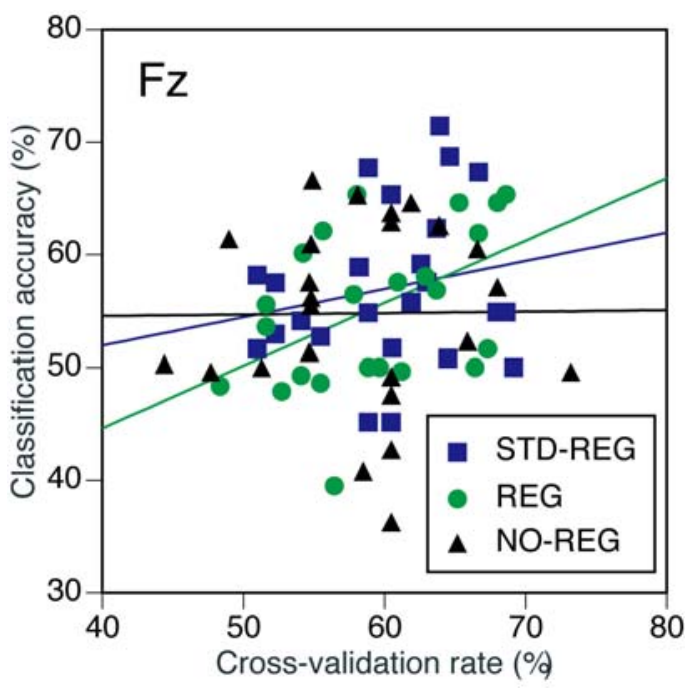

Figure 11. Relationship between crossvalidation rate and classification accuracy

(Fz)

figures, the deviation of data for Fz seems larger than the deviation for $\mathrm{Oz}$.

To confirm the relationship between the crossvalidation rate and classification accuracy, correlation coefficients (r) are calculated. These coefficients are summarized in Table 1 . In comparing the coefficients amongst the three procedures (STD-REG, REG and NO-REG), the coefficients for REG are significant and the highest for both $\mathrm{Oz}$ and Fz. This means that classification accuracy strongly reflects the cross-validation rate of the data when REG processing is applied. Therefore, REG is a more appropriate processing method for EEGs than STD-REG, though the results of STDREG processing are significant for some specific conditions.

\section{Conclusion}

To help a user's visual perception activity or to improve viewing conditions, new procedures for estimating viewed characters observed by users are

\section{Table 1. Correlation coefficients between cross-validation rate and classification accuracy}

\begin{tabular}{|c|c|c|c|}
\hline$r$ & STD-REG & REG & NO-REG \\
\hline $\mathrm{Oz}$ & 0.24 & 0.53 & 0.22 \\
\hline $\mathrm{Fz}$ & 0.19 & 0.47 & -.01 \\
\hline
\end{tabular}


proposed, using single channel and single-trial EEG waveforms from the frontal $(\mathrm{Fz})$ and occipital areas $(\mathrm{Oz})$ of human brain.

To improve estimation performance, a prediction technique was applied to observed EEG waveforms using Support Vector Regression (SVR) in order to formulate the relationship between single-trial EEG and ERP waveforms. Two processing procedures using the regression relationship were proposed. The first 20 trials for each group of symbols and Kanji were assigned to a training data set, and the remaining trials were assigned to a test data set. The estimation performance for the test data sets, such as cross validation rates and classification performance using features of the training data set, were compared between the three signal preparation conditions, and across perceptual processes from 150 to $800 \mathrm{msec}$.

As a result, the performance of cross validation rates of the test data set increased during the perceptual process for both $\mathrm{Fz}$ and $\mathrm{Oz}$ when predicted waveforms using the regression relationship between single-trial EEG and ERP waveforms were used. Also, the effectiveness of the prediction using the regression relationship for classification performance of viewed characters was determined during the perceptual process. This suggests that the procedure using the relationship between EEG and ERP is effective, and it is possible to conduct single-trial classifications of characters viewed by subjects using EEG waveforms.

Two signal processing procedures are proposed: REG for real time processing and STD-REG for post procedural processing. According to the relationship between the cross-validation rate and the classification accuracy, the REG procedure is the better one of the two.

\section{Future studies}

In this paper, the viewed characters were of two types: symbols and Kanji. If other kinds of characters can be predicted, wider application will be possible. Also, the first 20 trials were assigned to the training data set. The appropriate number of trials for the training data set should be determined. If only a low number of trials is appropriate for estimation and classification, the calibration procedure may become easier. This procedure has already been extended to EEG signals on multiple electrodes, and the preliminary performance of the classification is discussed [11]. To improve the performance, methods of extracting typical features from EEG waveforms are a common concern.

The procedure proposed in this paper for EEG signal processing is designed to apply to all subjects, as large differences in EEG signals exist between individuals. In this paper, some similarities in classification performance between one subject and the mean of all subjects provide the possibility of building a common model for calculations, such as in the relationship between ERP and EEG. If an appropriate model can be created using the data of some subjects, this model may be applied to other novel test subjects. This possibility should be examined to develop further applications for the classification of viewed characters.

These topics will also be subjects of our further study.

\section{Acknowledgements}

The authors appreciate the reviewer's comments about our conference paper and its selection by the iSociety 2010 Steering committee as a IJI journal article.

\section{References}

[1] H. Abe and M. Nakayama, "Event-related potential study of Kanji perception process", The Journal of the Institute of Image Information and Television Engineers, 60:397-404, 2006.

[2] H. Abe and M. Nakayama, "An investigation into Kanji character discrimination process from EEG signals", Enformatika, Transaction on Engineering, Computing and Technology, 18:13-18, 2006.

[3] B. Blankertz, G. Curio, and K. Müller, "Classifying single trial EEG: Towards brain computer interfacing”, In Proc. of Neural Information Processing Symposium (NIPS), pages 157-164, 2001.

[4] C.-C. Chang and C.-J. Lin. LIBSVM: a library for support vector machines, 2001. Software available at URL: http://www.csie.ntu.edu.tw/ cjlin/libsvm. (Access date 29 July 2009)

[5] R. Collobert. SVMTorch II. Software available at URL: http://www.idiap.ch/. (Access date 16 January 2008)

[6] R. Collobert and S. Bengio, "SVMTorch: Support vector machines for large-scale regression problems", Journal of Machine Learning Research, 1:143-160, 2001.

[7] D. Devlaminck, B.Wyns, L. Boullart, P. Sntens, and G. Otte, "Brain-Computer Interfaces: from theory to practice", In Proc. of 17th European Symposium on Artificial Neural Networks (ESANN), pp. 415-424, 2009.

[8] S. Makeig, M. Westerfield, T. P. Jung, J. T. J. Covington, T. J. Sejnowski, and E. Courchesne, "Functionally independent components of the late positive event-related potential during visual spatial attention”, Journal of Neuroscience, 19:2665-2680, 1999.

[9] Y. Miyawaki, H. Uchida, O. Yamashita, M. Sato, H. Morio, H. Tanabe, N. Sadato, and Y. Kamitani, "Visual image reconstruction from human brain activity using a combination of multiscale local image decoders", Neuron, 60:915-929, 2009. 
[10] M. Nakayama and H. Abe, "The possibility of singletrial classification of viewed characters using EEG waveforms", In Proc. of 17th European Symposium on Artificial Neural Networks (ESANN), pp. 449-454, 2009.

[11] M. Nakayama, H. Abe, "Performance of Single-trial Classifications of Viewed Characters using EEG Waveforms", International Journal of Cognitive Biometrics, in press.

[12] L. Parra, C. Alvino, A. Tang, B. Pearlmutter, N. Yeung, A. Osman, and P. Sajda, "Single-trial detection in EEG and MEG: Keeping it linear”, Neurocomputing, 5254:177-183, 2003.

[13] M. I. Posner and M. E. Raichle. Images of Mind. Scientific American Library, US, 1994.

[14] M. D. Rugg. Cognitive Neuroscience. Psychology Press, UK, 1997.

[15] Y. Shiba and H. Watabe. Toukeiteki Houhou II (Stastistical Method II). Shinyosha, Japan, 1976.

[16] A. J. Smola and B. Schölkopf, "A tutorial on support vector regression”, NeuroCOLT2 Technical Report Series, (NC2-TR-1998-030), October 1998. URL: http://www.neurocolt.com. (Access data 12 August 2004) 